\title{
Avaliação do Crescimento e do Custo da Alimentação do Pacu (Piaractus mesopotamicus Holmberg, 1887) Submetido a Ciclos Alternados de Restrição Alimentar e Realimentação
}

\author{
Valéria Leão Souza1, Elisabeth Criscuolo Urbinati², Maria Inez Espagnoli Geraldo Martins ${ }^{3}$, \\ Paulo César Silva ${ }^{4}$
}

\begin{abstract}
RESUMO - Avaliaram-se os efeitos da utilização de ciclos alternados de restrição alimentar e realimentação no crescimento do pacu, durante o período de engorda, e a viabilidade desta prática na produção comercial da espécie. Juvenis foram distribuídos em três tanques e submetidos a diferentes manejos alimentares, constituindo os tratamentos: A (alimentado ad libitum), B (restrição alimentar de 4 semanas, realimentado por 9 semanas) e C ( 6 semanas de restrição alimentar, realimentado por 7 semanas), em um total de 13 semanas por ciclo (4 ciclos experimentais). No final de cada ciclo alimentar, 20 peixes de cada tratamento foram amostrados e os dados biométricos registrados. Os valores obtidos para peso, comprimento total e fator de condição (K) foram submetidos a ANOVA e as médias comparadas pelo teste de Duncan. Os resultados mostraram que o tratamento C é o mais indicado somente para outono/inverno, promovendo maior crescimento, menor custo com ração, baixa conversão alimentar e maior receita líquida parcial. Entretanto, durante as estações mais quentes do ano (primavera/verão) outros programas de alimentação devem ser testados, utilizando-se períodos mais curtos de restrição alimentar. Quando se tratou da produção anual, o tratamento A respondeu melhor em termos de biomassa produzida, apesar do maior gasto com alimentação, visto que, com o aumento da temperatura, o crescimento dos peixes dos demais tratamentos ficou prejudicado.
\end{abstract}

Palavras-chave: avaliação econômica, ciclos de restrição alimentar e realimentação, crescimento, custos com alimentação

\section{Evaluation of the Growth and Feeding Costs of Pacu (Piaractus mesopotamicus) Submitted to Alternate Cycles of Feeding Restriction and Refeeding}

\begin{abstract}
The present study evaluated the effects of alternating food restriction and refeeding cycles on the growth of pacu, during the growout phase and the viability of this practice in the commercial production of the species. Juvenile fish were distributed in 3 tanks and submitted to different feeding strategies: group A (fed ad libitum daily), B (food restricted to 4 weeks and refed for 9 weeks) and C (food restricted to 6 weeks and refed for 7 weeks) totalizing 13 weeks per cycle (4 experimental cycles). At the end of each feeding cycle, 20 fish per treatment were sampled and the biometric data were registered. Values of weight, total length and condition factor $(\mathrm{K})$ were submitted to ANOVA and the averages were compared by Duncan test. The results showed that the treatment $\mathrm{C}$ presented better results only in autumn/winter periods, promoting better growth, lower cost ration, lower food conversion and better partial net income. Otherwise, during the warmer periods of the year (spring/summer) other feeding strategies must be tested, utilizing shorter periods of food restriction. Regarding to the annual production, the treatment A showed higher biomass, although higher feeding costs. With the increase in the temperature, the growth of the fish from the other treatments was delayed.
\end{abstract}

Key Words: economic analysis, food restriction and refeeding cycle, growth, feeding costs

\section{Introdução}

O pacu, Piaractus mesopotamicus, é um dos peixes de água doce mais estudados nas regiões Sul, Sudeste e Centro-Oeste do Brasil. Originário da Bacia do Rio Prata e do Pantanal do Mato Grosso, é morador habitual das grandes coleções de água, como as dos rios Paraná, Mogi-guaçu, Paraíba e baixo Tietê (Petreri Jr., 1989). Esta espécie é migratória, onívora, mas apresenta um hábito alimentar especificamente frugívoro-herbívoro, do tipo podador e de caráter oportunista (Silva, 1985). Suas características de precocidade, rusticidade, carne saborosa e de alto valor comercial, além do ótimo crescimento e

\footnotetext{
* Autor para correspondência.

1 Zootecnista, Dra., Bolsista do CNPq - DCR-2C. Depto. de Produção Animal. Escola de Veterinária da Universidade Federal de Goiás - EV/UFG, Goiânia, Goiás. E.mail: vleaosouza@hotmail.com

2 Biomédica, PhD., Centro de Aqüicultura da Universidade Estadual Paulista - CAUNESP, Via de Acesso Prof. Paulo Donato Castelane s/n, Jaboticabal, São Paulo, 14884-900, Brasil. E.mail: bethurb@caunesp.unesp.br

3 Médica Veterinária, Dra., Depto. de Economia Rural. Faculdade de Ciências Agrárias e Veterinárias - FCAV/UNESP, Campus de Jaboticabal. Jaboticabal, São Paulo. E.mail: minezesp@fcav.unesp.br

4 Médico Veterinário, Dr., Depto. de Produção Animal. Escola de Veterinária da Universidade Federal de Goiás - EV/UFG, Goiânia, Goiás. E.mail: pcsilva@vet.ufg.br
} 
adaptação à alimentação artificial propiciam o sucesso da criação em sistemas de cultivo intensivo (Castagnoli \& Cyrino, 1986).

Trabalhos realizados com esta espécie nas regiões Sul (Borghetti \& Canzi, 1993), Sudeste (Bernardino \& Ferrari, 1989; Ferrari et al., 1990; Angelini et al., 1992) e Centro-Oeste (Ferraz de Lima et al., 1988; Chabalin \& Ferraz de Lima, 1988) do Brasil evidenciaram uma relação entre crescimento e temperatura, com desaceleração no ritmo de crescimento, durante as estações de outono e inverno. O papel da temperatura na regulação da alimentação e crescimento é pouco descrito em peixes, mas sabe-se que a maioria deles se alimenta melhor (Paul et al., 1995) e as taxas de crescimento tendem a ser mais altas (Dobson \& Holmes, 1984), na primavera e verão.

As flutuações temporal e espacial de alimento no ambiente aquático, ou a migração para desova, submetem os peixes a períodos naturais de privação alimentar (Wieser et al., 1992; Mehner \& Wieser, 1994). Em condições experimentais, estudos (Blasco et al., 1991; Kieffer \& Tufts, 1998) têm revelado que a restrição envolve complexas alterações fisiológicas e metabólicas para promover o ajuste biológico do animal, e suas conseqüências finais são altamente dependentes da espécie considerada, da idade do peixe e das condições experimentais como temperatura da água, fotoperíodo, dieta pré-jejum e duração do período de jejum.

Enquanto juvenis de pacu (Piaractus mesopotamicus) mantiveram o peso corporal estável, após 60 dias de restrição alimentar, tanto em épocas de temperaturas mais altas $\left(26,0^{\circ} \mathrm{C}\right)$ (Souza, $1998)$ quanto em mais baixas $\left(23,6^{\circ} \mathrm{C}\right)$ (Souza et al., 2000), Clarias gariepinus apresentou perda de peso em período de jejum e temperatura semelhantes (Zamal \& Ollevier, 1995). Perdas também foram registradas para Acipenser transmontanus (Hung et al., 1997) e Salmo salar (Einen et al., 1998) em períodos mais longos a temperaturas mais baixas. Nenhuma alteração no peso foi observada para Oncorhyncus mykiss, durante 5 dias, a $15^{\circ} \mathrm{C}$ (Figueroa et al., 2000). Já a tilápia (Oreochromis mossambicus x O. niloticus) perdeu peso, a $25,6^{\circ} \mathrm{C}$, em um período de 30 dias (Wang et al., 2000).

$\mathrm{O}$ retorno às condições adequadas de alimentação, após um período de inanição ou má nutrição, promove um crescimento rápido no animal, chamado crescimento compensatório (Dobson \& Holmes, 1984; Paul et al., 1995; Xie et al., 2001). O crescimento compensatório em peixes pode ter importante aplica- ção na aqüicultura (Quinton \& Blake 1990; Hayward et al., 1997). Uma exploração adequada deste fenômeno pode resultar em melhor produção, com aumento na taxa de crescimento e eficiência alimentar e redução do custo com alimentação (Wang et al., 2000; Maclean \& Metcalfe, 2001).

A maioria dos estudos enfatizando o crescimento compensatório utiliza espécies de água fria e são poucos os relatos com espécies de água quente (Souza et al., 2000; Sirol et al., 2000; Wang et al., 2000; Xie et al., 2001). Além disso, foram realizados apenas períodos de privação alimentar seguidos de realimentação para demonstrar o crescimento compensatório (Souza et al., 2000; Sirol et al., 2000; Qian et al., 2000). Alguns estudos têm avaliado ciclos repetidos de restrição alimentar e realimentação em relação ao crescimento compensatório (Mélard et al., 1997; Ali \& Wootton, 2001; Hayward \& Wang, 2001; Chatakondi \& Yant, 2001).

O objetivo deste experimento foi avaliar os efeitos da utilização de ciclos alternados de restrição alimentar e realimentação no crescimento do pacu (Piaractus mesopotamicus), durante a fase de engorda, e a viabilidade desta prática como procedimento de rotina na produção comercial desta espécie e alternativa para diminuir os custos com alimentação.

\section{Material e Métodos}

O experimento foi conduzido no Setor de Piscicultura da Escola de Veterinária da Universidade Federal de Goiás - UFG, Goiânia, GO (16\%41' de latitude sul, $49^{\circ} 17^{\prime}$ de longitude oeste e a $730 \mathrm{~m}$ de altitude), de 23 de março de 1999 a 21 de março de 2000 (52 semanas).

Foram utilizados juvenis de pacu (Piaractus mesopotamicus), com peso médio inicial de $83,5 \pm 17,1$ g e comprimento total de $16,5 \pm 1,2 \mathrm{~cm}$. Os animais foram divididos em três tratamentos: A - controle, alimentado ad libitum, diariamente; B - 4 semanas de restrição alimentar, 9 semanas de realimentação; C - 6 semanas de restrição alimentar, 7 semanas de realimentação, e distribuídos em três tanques (respectivamente para os tratamentos A, B e C) contendo, inicialmente, 169 animais cada.

Cada 13 semanas de restrição alimentar/realimentação constituíam um ciclo alimentar. Assim, os peixes foram submetidos a quatro ciclos alimentares durante o ano, que começavam com restrição alimentar e terminavam com realimentação. 
Ostanques de $50 \mathrm{~m}^{2}$, profundidade média de $1,10 \mathrm{~m}$, vazão inicial de $3 \mathrm{~L} / \mathrm{min}$. e final de $6 \mathrm{~L} / \mathrm{min}$., abastecidos com água procedente de uma represa à montante, foram previamente preparados com $100 \mathrm{~g} / \mathrm{m}^{2}$ de calcário agrícola e $200 \mathrm{~g} / \mathrm{m}^{2}$ de esterco de galinha.

Antes de iniciar o período experimental, os animais passaram por duas semanas de adaptação nos tanques, recebendo ração comercial extrusada diariamente (duas vezes ao dia - 9 e $16 \mathrm{~h}$ ), contendo $28 \%$ de proteína bruta, fornecida a lanço, oferecida $a d$ libitum. Após este período, os animais do grupo A continuaram sendo alimentados diariamente e aqueles dos grupos B e C passaram pelos respectivos ciclos alimentares. Durante a realimentação, o arraçoamento foi realizado, conforme indicado anteriormente, e a quantidade de alimento ofertado, anotada.

Ao final de cada ciclo (13 semanas), 20 peixes de cada tanque foram capturados ao acaso, pesados e medidos individualmente, para estimar o peso e comprimento total médio e fator de condição $\left[\mathrm{FC}=\mathrm{P} / \mathrm{Ct}^{\mathrm{b}}\right.$, em que: $\mathrm{P}$ é o peso corporal (g), Ct é o comprimento total $(\mathrm{cm})$ e b é estimado pela equação da relação peso/comprimento $\left(\mathrm{y}=\mathrm{ax}^{\mathrm{b}}\right)$, após transformação logarítmica e ajuste pelo método dos mínimos quadrados dos dados (Ricker, 1975)]. Estes dados foram avaliados por análise de variância (ANOVA) e tiveram suas médias comparadas pelo teste de Duncan (Banzatto \& Kronka, 1989).

A taxa de crescimento específico [TCE $=(\ln$ Pf

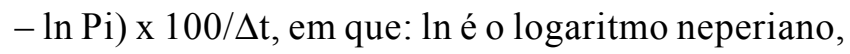
Pf e Pi são os pesos médios finais e iniciais ( $g$ ) dos peixes e $\Delta \mathrm{t}$, o intervalo de tempo (dias) entre as pesagens (Weatherley \& Gill, 1987)], a conversão alimentar aparente $[\mathrm{CAA}=\mathrm{Qr} /(\mathrm{Bf}-\mathrm{Bi})$, em que: $\mathrm{Qr}$ é a quantidade de ração fornecida $(\mathrm{kg})$ e $\mathrm{Bf}$ e $\mathrm{Bi}$, as biomassas iniciais e finais $(\mathrm{kg})$ (Bernardino \& Ferrari, 1989)] e a eficiência de crescimento $[\mathrm{EC}=(\mathrm{Bf}-\mathrm{Bi}) /$ Qr (Hayward et al., 2000)] foram calculados com base nos valores médios de cada tratamento.

Avaliou-se economicamente o experimento, considerando-se para os três tratamentos, nos quatro ciclos, apenas o custo da alimentação, que foi o fator variável. Neste sentido, os outros fatores geradores de custo foram entendidos como constantes e iguais para as situações analisadas e não foi objeto de análise. Portanto, no custo da alimentação considerou-se o gasto com ração e o custo da mão-de-obra, calculado da seguinte forma: custo da ração [CR $=$ Qr x Pr, em que: Pr é o preço $(\mathrm{R} \$$ ) do quilo da ração (cotado a $\mathrm{R} \$ 0,64$ no final do experimento)], custo com alimentação $[\mathrm{CA}=\mathrm{CR}+$
(HMO x CH), onde: HMO é o número de horas de mãode-obra gasta para alimentar os três tanques (estimado em 1 hora/dia) e $\mathrm{CH}$, o custo da hora (calculada com base no salário mínimo vigente em março/2000 (R \$130,00 $+31 \%$ de encargos sociais diretos/24 dias úteis $\mathrm{x} 8$ horas/ dia $=\mathrm{R} \$ 0,89$ a hora))]. Calculou-se a receita bruta [RB = B x Pp, em que: B é a biomassa (kg) e Pp, o preço (R\$) do quilograma do peixe vivo, cotado a $R \$ 2,20 / \mathrm{kg}$ ] e a receita líquida parcial [RLP $=\mathrm{RB}-\mathrm{CA}]$. Quando os valores foram projetados para $1 \mathrm{ha}$, considerou-se um número de horas para alimentação de 4 horas/dia.

A temperatura da água foi monitorada diariamente $(9$ e $16 \mathrm{~h}$ ) e, quinzenalmente, amostras de água dos tanques foram coletadas (7h30) para obtenção dos valores de oxigênio dissolvido, $\mathrm{pH}$, alcalinidade total, amônia, nitritoe transparência utilizando-se um kit comercial.

\section{Resultados e Discussão}

\section{Qualidade da água}

Na Tabela 1 são apresentados os valores médios mensais dos parâmetros físicos e químicos da água e, na Figura 1, as oscilações na temperatura, durante o período de estudo.

Os níveis de oxigênio dissolvido começaram a diminuir a partir de agosto. Em função disso, do aumento nos valores de amônia e da elevação da temperatura, aumentou-se gradativamente a vazão da água nos tanques até o final do período experimental. Apesar de serem

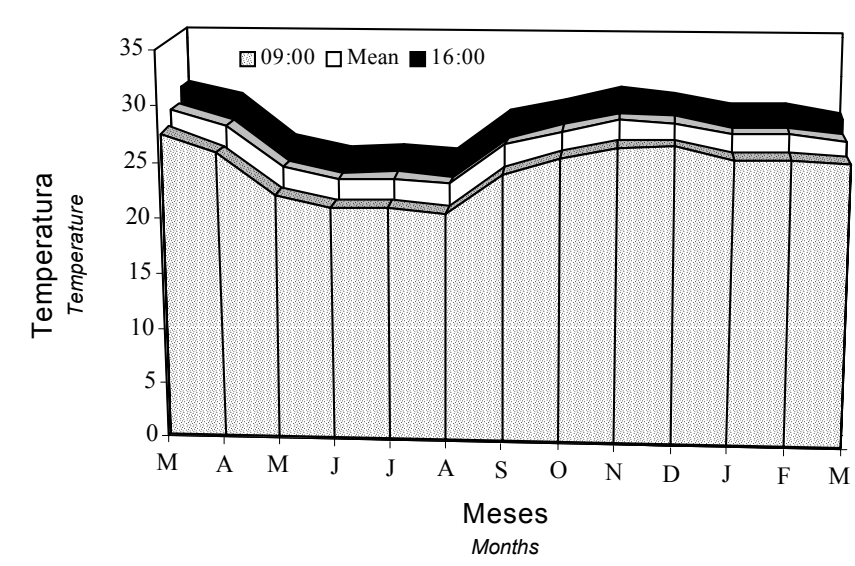

Figura 1 - Valores médios mensais da temperatura da água dos tanques, durante o período de estudo.

Figure 1 - Mean water temperature values in the tanks, during the experimental period.

R. Bras. Zootec., v.32, n.1, p.19-28, 2003 
Tabela 1 - Valores médios mensais de oxigênio dissolvido $\left(\mathrm{O}_{2} \mathrm{D}\right)$, pH, alcalinidade total (AT), amônia, nitrito e transparência (Tp) da água dos tanques (1 - Tratamento A; 2 - Tratamento B; 3 - Tratamento $C$ ) e da caixa de distribuição (CD), durante o período de estudo

Table 1 - Mean values of the water dissolved oxygen $\left(\mathrm{DO}_{2}\right), \mathrm{pH}$, total alkalinity (TA), ammonia, nitrite and transparency (Tp) in the tanks (1- Treatment A; 2 - Treatment B; 3 - Treatment C) and distribution source (DS), during the experimental period

\begin{tabular}{|c|c|c|c|c|c|c|c|c|c|c|c|c|c|c|}
\hline \multirow[b]{2}{*}{$\begin{array}{l}\text { Variáveis } \\
\text { Variables }\end{array}$} & \multirow[b]{2}{*}{$\begin{array}{c}\text { Tanque } \\
\text { Tank }\end{array}$} & \multicolumn{13}{|c|}{$\begin{array}{l}\text { Meses } \\
\text { Months }\end{array}$} \\
\hline & & M & A & M & $\mathrm{J}$ & $\mathrm{J}$ & $\mathrm{A}$ & $\mathrm{S}$ & $\mathrm{O}$ & $\mathrm{N}$ & $\mathrm{D}$ & $\mathrm{J}$ & $\mathrm{F}$ & $\mathrm{M}$ \\
\hline $\mathrm{O} \mathrm{O}_{2}$ & 1 & - & 5,60 & 6,90 & 6,70 & 6,40 & 2,20 & 4,50 & 5,00 & 4,00 & 3,80 & 1,80 & 1,90 & 1,50 \\
\hline $\mathrm{DO}_{2}^{2}$ & 2 & - & 5,70 & 5,70 & 5,90 & 7,00 & 4,90 & 4,40 & 4,70 & 1,50 & 0,80 & 2,70 & 1,20 & 1,20 \\
\hline \multirow[t]{2}{*}{$(\mathrm{mg} / \mathrm{L})$} & 3 & - & 6,00 & 6,60 & 6,80 & 7,30 & 5,70 & 7,00 & 5,40 & 6,30 & 2,50 & 4,40 & 3,30 & 1,50 \\
\hline & $\mathrm{CD}$ & - & 7,00 & 7,40 & 7,80 & 7,80 & 8,30 & 7,60 & 6,80 & 6,50 & 6,60 & 5,80 & 6,30 & 6,10 \\
\hline \multirow[t]{4}{*}{$\mathrm{pH}$} & 1 & 7,30 & 7,00 & 7,20 & 7,30 & 7,20 & 7,10 & 7,20 & 7,30 & 6,90 & 7,20 & 6,90 & 6,70 & 6,70 \\
\hline & 2 & 7,00 & 7,00 & 7,10 & 7,10 & 7,20 & 7,30 & 7,10 & 7,20 & 6,80 & 6,70 & 6,90 & 6,60 & 6,70 \\
\hline & 3 & 7,00 & 7,00 & 7,20 & 7,30 & 7,40 & 7,30 & 7,40 & 7,30 & 7,00 & 6,80 & 7,00 & 6,70 & 6,60 \\
\hline & $\mathrm{CD}$ & 7,00 & 6,90 & 6,90 & 7,30 & 7,10 & 7,50 & 6,80 & 7,10 & 6,80 & 7,10 & 6,90 & 6,80 & 6,80 \\
\hline $\mathrm{AT}$ & 1 & 28,00 & 26,00 & 26,00 & 20,00 & 20,00 & 42,00 & 34,00 & 31,00 & 30,00 & 34,00 & 29,00 & 29,00 & 29,00 \\
\hline$T A$ & 2 & 24,00 & 30,00 & 25,00 & 23,00 & 21,00 & 30,00 & 32,00 & 26,00 & 40,00 & 31,00 & 28,00 & 29,00 & 29,00 \\
\hline (mg/L & 3 & 24,00 & 28,00 & 26,00 & 24,00 & 26,00 & 31,00 & 30,00 & 30,00 & 30,00 & 31,00 & 30,00 & 23,00 & 29,00 \\
\hline $\left.\mathrm{HCO}_{3}\right)$ & $\mathrm{CD}$ & 24,00 & 26,00 & 23,00 & 22,00 & 24,00 & 26,00 & 35,00 & 20,00 & 20,00 & 28,00 & 27,00 & 38,00 & 26,00 \\
\hline Amônia & 1 & 0,00 & 0,03 & 0,04 & 0,01 & 0,06 & 0,17 & 0,15 & 0,12 & 0,06 & 0,04 & 0,06 & 0,24 & 0,27 \\
\hline Ammonia & 2 & 0,00 & 0,03 & 0,06 & 0,01 & 0,06 & 0,09 & 0,21 & 0,11 & 0,06 & 0,25 & 0,05 & 0,26 & 0,63 \\
\hline$(\mathrm{mg} / \mathrm{L}$ & 3 & 0,00 & 0,03 & 0,05 & 0,04 & 0,06 & 0,06 & 0,12 & 0,11 & 0,06 & 0,06 & 0,05 & 0,22 & 0,24 \\
\hline $\left.\mathrm{N}-\mathrm{NH}_{3}\right)$ & $\mathrm{CD}$ & 0,00 & 0,03 & 0,01 & 0,01 & 0,03 & 0,04 & 0,07 & 0,08 & 0,03 & 0,02 & 0,05 & 0,16 & 0,16 \\
\hline Nitrito & 1 & 0,00 & 0,01 & 0,00 & 0,01 & 0,01 & 0,01 & 0,01 & 0,01 & 0,01 & 0,01 & 0,00 & 0,02 & 0,02 \\
\hline Nitrite & 2 & 0,00 & 0,01 & 0,00 & 0,01 & 0,01 & 0,01 & 0,01 & 0,02 & 0,01 & 0,02 & 0,00 & 0,02 & 0,02 \\
\hline (mg/L & 3 & 0,00 & 0,01 & 0,00 & 0,01 & 0,01 & 0,01 & 0,01 & 0,02 & 0,01 & 0,02 & 0,01 & 0,02 & 0,02 \\
\hline $\mathrm{N}-\mathrm{NO}_{2}$ ) & $\mathrm{CD}$ & 0,00 & 0,01 & 0,00 & 0,01 & 0,01 & 0,01 & 0,01 & 0,01 & 0,00 & 0,00 & 0,00 & 0,02 & 0,01 \\
\hline $\mathrm{Tp}$ & 1 & 82,50 & 77,80 & 81,80 & 84,30 & 79,30 & 84,50 & 70,00 & 78,80 & 54,50 & 47,50 & 58,50 & 50,00 & 73,70 \\
\hline \multirow[t]{2}{*}{$(\mathrm{cm})$} & 2 & 84,00 & 80,00 & 73,50 & 75,00 & 60,00 & 86,00 & 85,70 & 87,50 & 83,80 & 87,00 & 84,00 & 78,00 & 79,30 \\
\hline & 3 & 82,50 & 92,80 & 94,30 & 97,30 & 82,30 & 90,80 & 66,00 & 95,30 & 53,00 & 79,00 & 96,00 & 83,00 & 95,30 \\
\hline
\end{tabular}

efetuadas adubações mensais nos tanques, a transparência da água manteve-se alta durante quase todo o período de estudo. Já os valores de $\mathrm{pH}$, nitrito e alcalinidade total mantiveram-se dentro da faixa limite para o cultivo do pacu.

Os valores encontrados para os parâmetros físicos e químicos da água foram considerados adequados e/ou tolerados pelo pacu, pois segundo Sipaúba-Tavares (1994) e Ostrensky \& Boeger (1998), valores de oxigênio dissolvido entre 4 e $6 \mathrm{mg} / \mathrm{L}$, pH de 6 a 8 , transparência de 25 a $45 \mathrm{~cm}$ e alcalinidade total acima de $20 \mathrm{mg} / \mathrm{L}$ são ideais para o cultivo da espécie. A faixa de tolerância da amônia situa-se entre 0,6 a 2,0 mg/L e para nitrito, até 0,5 mg/L (Sipaúba-Tavares, 1994). Já o valor mínimo de $\mathrm{O}_{2} \mathrm{D}$ suportado pelo pacu é de $1,5 \mathrm{mg} / \mathrm{L}$ e os limites de temperatura para a sua criação são de 20 e $30^{\circ} \mathrm{C}$ (Ostrensky \& Boeger 1998).

\section{Índices zootécnicos}

Não foram observadas diferenças significativas $(\mathrm{P}>0,05)$ para peso, comprimento total e fator de condição no início e durante o $1^{\circ}$ ciclo alimentar (Tabela 2). Entretanto, no $2^{\circ}$ ciclo, apesar das diferenças não serem significativas $(\mathrm{P}>0,05)$ para peso corporal, observa-se que as médias dos tratamentos $\mathrm{B}$ e $\mathrm{C}$ foram superiores às de $\mathrm{A}$, apresentando uma possível resposta compensatória. $\mathrm{O}$ fator de condição reforçou $(\mathrm{P}<0,05)$ esta possibilidade, pois através dele pode-se acompanhar o grau de atividade alimentar de uma espécie, verificando se ela está ou não fazendo bom uso da fonte nutricional (Weatherley \& Gill, 1987). Além disso, a eficiência de crescimento foi mais acentuada para os peixes do tratamento $C$, neste período (Figura 2).

Durante o outono e inverno ( $1^{\circ}$ e $2^{\circ}$ ciclos), o crescimento do pacu, tanto em peso quanto em

\section{R. Bras. Zootec., v.32, n.1, p.19-28, 2003}


Tabela 2 - Valores médios ( $\pm D P$ ) obtidos para peso corporal $(P C)$, comprimento total $(C T)$ e fator de condição (FC) dos peixes, no início (Dia 0) e em cada ciclo alimentar

Table 2 - Mean values $( \pm D P)$ of body weight (BW), total length (TL) and condition factor (CF) of fish in the beginning of the experiment (Day 0) and at each feeding cycle

\begin{tabular}{|c|c|c|c|c|}
\hline \multirow{2}{*}{ 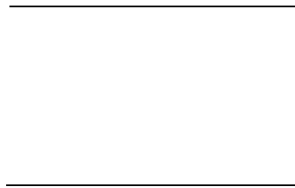 } & \multirow{2}{*}{$\begin{array}{l}\text { Tratamentos* } \\
\text { Treatments* }\end{array}$} & \multicolumn{3}{|c|}{$\begin{array}{l}\text { Variáveis } \\
\text { Variables }\end{array}$} \\
\hline & & $\begin{array}{l}\mathrm{PC}(\mathrm{g}) \\
B W(g)\end{array}$ & $\begin{array}{l}\mathrm{CT}(\mathrm{cm}) \\
T C(\mathrm{~cm})\end{array}$ & $\begin{array}{l}\mathrm{FC} \\
C F\end{array}$ \\
\hline $\begin{array}{l}\text { Dia 0 } \\
\text { Day 0 } \\
(23 / 03 / 99)\end{array}$ & $\begin{array}{l}\text { A } \\
\mathrm{B} \\
\mathrm{C}\end{array}$ & $\begin{array}{l}83,4 \mathrm{a}( \pm 13,7) \\
82,3 \mathrm{a}( \pm 17,7) \\
84,9 \mathrm{a}( \pm 20,1)\end{array}$ & $\begin{array}{l}16,4 \mathrm{a}( \pm 0,9) \\
16,2 \mathrm{a}( \pm 1,1) \\
16,9 \mathrm{a}( \pm 1,4)\end{array}$ & $\begin{array}{l}0,0258 \mathrm{a}( \pm 0,001) \\
0,0265 \mathrm{a}( \pm 0,001) \\
0,0263 \mathrm{a}( \pm 0,002)\end{array}$ \\
\hline $\begin{array}{l}1^{\mathrm{o}} \text { ciclo } \\
1^{\text {st }} \text { cycle } \\
(23 / 03 \mathrm{a} 22 / 06 / 99)\end{array}$ & $\begin{array}{l}\mathrm{A} \\
\mathrm{B} \\
\mathrm{C}\end{array}$ & $\begin{array}{l}120,7 \mathrm{a}( \pm 33,3) \\
120,9 \mathrm{a}( \pm 35,9) \\
121,9 \mathrm{a}( \pm 40,7)\end{array}$ & $\begin{array}{l}18,1 \mathrm{a}( \pm 1,6) \\
18,8 \mathrm{a}( \pm 2,0) \\
18,6 \mathrm{a}( \pm 2,0)\end{array}$ & $\begin{array}{l}0,0281 \mathrm{a}( \pm 0,003) \\
0,0285 \mathrm{a}( \pm 0,002) \\
0,0282 \mathrm{a}( \pm 0,003)\end{array}$ \\
\hline $\begin{array}{l}2^{\circ} \text { ciclo } \\
2^{\text {nd }} \text { cycle } \\
(23 / 06 \text { a } 21 / 09 / 99)\end{array}$ & $\begin{array}{l}\mathrm{A} \\
\mathrm{B} \\
\mathrm{C}\end{array}$ & $\begin{array}{l}158,5 \mathrm{a}( \pm 42,9) \\
163,4 \mathrm{a}( \pm 61,3) \\
184,2 \mathrm{a}( \pm 52,4)\end{array}$ & $\begin{array}{l}19,4 \mathrm{a}( \pm 1,5) \\
19,5 \mathrm{a}( \pm 2,5) \\
20,2 \mathrm{a}( \pm 2,0)\end{array}$ & $\begin{array}{c}0,0273 \mathrm{~b}( \pm 0,002) \\
0,0298 \mathrm{a}( \pm 0,002) \\
0,0294 \mathrm{a}( \pm 0,002)\end{array}$ \\
\hline $\begin{array}{l}3^{\circ} \text { ciclo } \\
3^{r d} \text { cycle } \\
(22 / 09 \text { a } 21 / 12 / 99)\end{array}$ & $\begin{array}{l}\text { A } \\
\text { B } \\
\text { C }\end{array}$ & $\begin{array}{c}556,5 \mathrm{a}( \pm 133,1) \\
456,3 \mathrm{~b}( \pm 83,1) \\
449,2 \mathrm{~b}( \pm 88,9)\end{array}$ & $\begin{array}{l}30,6 \mathrm{a}( \pm 2,4) \\
27,9 \mathrm{~b}( \pm 1,6) \\
27,8 \mathrm{~b}( \pm 1,8)\end{array}$ & $\begin{array}{l}0,0298 \mathrm{a}( \pm 0,002) \\
0,0272 \mathrm{~b}( \pm 0,001) \\
0,0268 \mathrm{~b}( \pm 0,001)\end{array}$ \\
\hline $\begin{array}{l}4^{\circ} \text { ciclo } \\
4^{\text {th }} \text { cycle } \\
(22 / 12 / 99 \mathrm{a} 21 / 03 / 00)\end{array}$ & $\begin{array}{l}\mathrm{A} \\
\mathrm{B} \\
\mathrm{C}\end{array}$ & $\begin{array}{c}1118,8 \mathrm{a}( \pm 293,6) \\
809,5 \mathrm{~b}( \pm 181,6) \\
652,3 \mathrm{c}( \pm 148,9)\end{array}$ & $\begin{array}{l}36,6 \mathrm{a}( \pm 2,9) \\
32,8 \mathrm{~b}( \pm 2,3) \\
30,7 \mathrm{c}( \pm 2,2)\end{array}$ & $\begin{array}{c}0,0303 \mathrm{a}( \pm 0,002) \\
0,0242 \mathrm{~b}( \pm 0,002) \\
0,0191 \mathrm{c}( \pm 0,001)\end{array}$ \\
\hline
\end{tabular}

* A [controle, alimentado ad libitum, diariamente]; B [4 semanas de restrição alimentar/9 semanas de realimentação]; C [6 semanas de restrição alimentar/7 semanas de realimentação].

Médias seguidas de mesma letra na coluna, dentro de cada ciclo, não diferem entre si pelo teste Duncan $(P>0,05)$.

* A [control, fed daily ad libitum]; B [4 weeks of feeding restriction/9 weeks of refeeding]; C [6 weeks of feeding restriction/7 weeks of refeeding]. Means followed by the same letter in the column within each cycle are not different by Duncan test $(P>0.05)$.

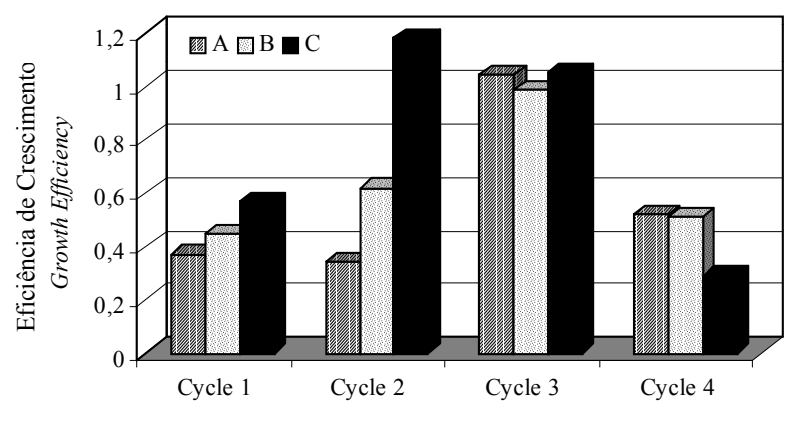

Figura 2 - Eficiência de crescimento dos peixes por tratamento (A-alimentado ad libitum, diariamente; B - 4 semanas de restrição alimentar/9 semanas de realimentação; C - 6 semanas de restrição alimentar/7 semanas de realimentação), em cada ciclo alimentar.

Figure 2 - Fish growth efficiency in the treatments (A - control, fed daily ad libitum; $B$ - 4 weeks of feeding restriction/ 9 weeks of refeeding; C - 6 weeks of feeding restriction/7 weeks of refeeding, in each cycle.

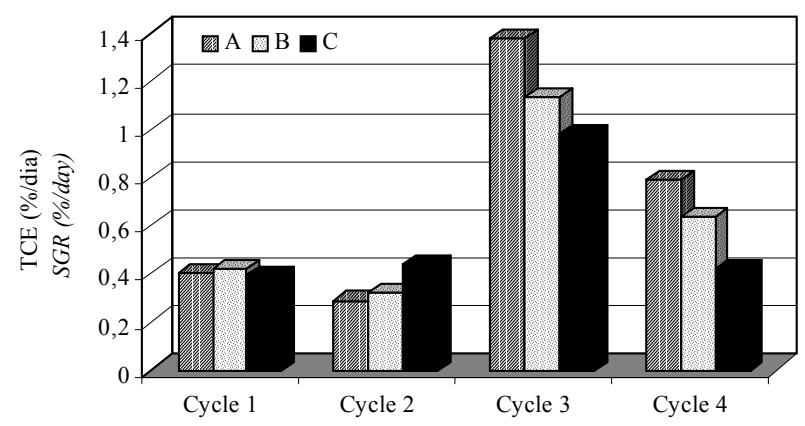

Figura 3 - Taxa de crescimento específico dos peixes por tratamento (A - alimentado ad libitum, diariamente; B - 4 semanas de restrição alimentar/9 semanas de realimentação; C- 6 semanas de restrição alimentar/7 semanas de realimentação), em cada ciclo alimentar.

Figure 3 - Specific growth rate of the fish in the treatments $(A-$ control, fed daily ad libitum; $B$ - 4 weeks of feeding restriction/9 weeks of refeeding; $C$ - 6 weeks of feeding restriction/7 weeks of refeeding, in each cycle. 
comprimento, foi menor, com menores taxas de crescimento específico (Figura 3). Respostas semelhantes foram obtidas por Ferraz de Lima et al. (1988), que evidenciaram, para a mesma espécie, a existência de uma relação de causa e efeito entre os índices de crescimento específico e as variações na temperatura, com ocorrência de índices reduzidos nos períodos mais frios do ano.

Segundo a literatura, o crescimento compensatório pode ser obtido por hiperfagia (Wang et al., 2000; Xie et al., 2001) ou de uma combinação de hiperfagia e aumento na eficiência de crescimento (Jobling et al., 1993; Hayward et al., 1997; Qian et al., 2000). Neste estudo, a quantidade de ração consumida e acumulada por cada grupo de peixes em diferentes ciclos foi computada para elucidar possíveis aumentos na ingestão de alimento durante a realimentação (Figura 4). Considerando cada ciclo de 13 semanas, as quatro primeiras semanas corresponderam ao período em que os peixes dos tratamentos $\mathrm{BeC}$ encontravam-se em restrição alimentar e somente os do A consumiram ração. Nas duas semanas subseqüentes registrou-se a ingestão de alimento dos peixes do tratamento $\mathrm{B}$, quando realimentados, após a privação em relação ao mesmo período de consumo do A e, nas sete semanas restantes, registrou-se o consumo

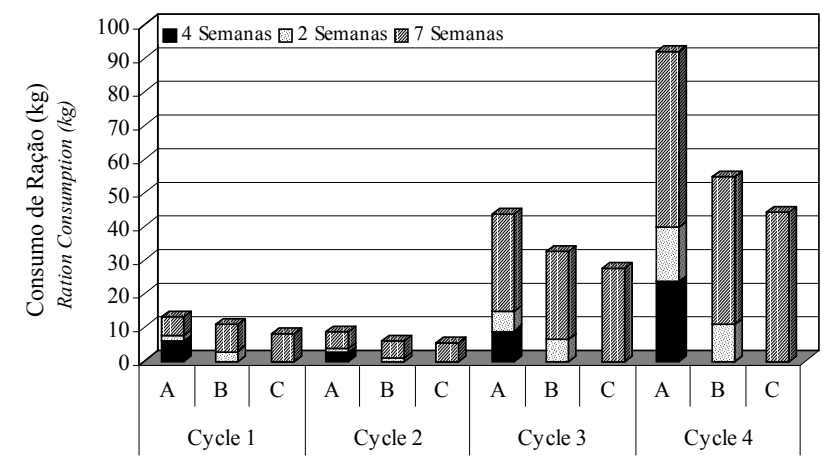

Figura 4 - Ração consumida pelo pacu, durante as fases de alimentação (semanas), nos diferentes tratamentos (A - alimentado ad libitum, diariamente; B - 4 semanas de restrição alimentar/9 semanas de realimentação; C - 6 semanas de restrição alimentar/7 semanas de realimentação), em cada ciclo alimentar

Figure 4 - Consumed ration by pacu, during the feeding phases (weeks), in the treatments ( $A$ - control, fed daily ad libitum; $B$ - 4 weeks of feeding restriction/9 weeks of refeeding; C - 6 weeks of feeding restriction/7 weeks of refeeding, in each cycle. de ração quando os peixes de todos os tratamentos estavam se alimentando. Com isso, observou-se, no $1^{\circ}$ ciclo, que os peixes dos tratamentos $\mathrm{C}$ e $\mathrm{B}$ consumiram mais alimento ( 8,38 e $8,24 \mathrm{~kg}$, respectivamente) nos períodos de realimentação do que os do $\mathrm{A}(5,47 \mathrm{~kg})$, no mesmo período (Figura 4).

Melhor eficiência da produção pode ser obtida no inverno, através de regimes alimentares que incluem restrição, pois, nessa época, com a queda da temperatura, o metabolismo, a ingestão de alimento, a digestão e a resposta imune dos peixes diminuem (Sealey et al., 1998). Ciclos de 3 semanas de jejum e 3 semanas de realimentação resultaram em melhor peso, comprimento e taxa de crescimento específico em Oncorhynchus mykiss $\left(12\right.$ a $\left.14^{\circ} \mathrm{C}\right)$ do que 1 semana de jejum e 1 semana de realimentação, ou 2 e 2 semanas, e a sua produção foi semelhante a dos peixes constantemente alimentados, durante um ou dois ciclos completos (Quinton \& Blake, 1990). Ictalurus punctatus, com pesos iniciais de 40,8 e $680,4 \mathrm{~g}$, continuamente e parcialmente restritos $(60 \%$ da alimentação dos controles), alcançaram o mesmo peso daqueles continuamente alimentados, apresentando ganho de peso compensatório quando realimentados na primavera (Sealey et al., 1998). Em Perca flavescens, a $21^{\circ} \mathrm{C}$, ocorreu hiperfagia e crescimento compensatório quando foram utilizados ciclos de 12, 17 e 22 dias de jejum, com subseqüente realimentação, até o desaparecimento da hiperfagia, num estudo de 125 dias. A $17^{\circ} \mathrm{C}$, ciclos alternados de 12 dias de alimentação com ração apenas para manutenção $(0,5$ a $1 \%$ do peso corporal) e posterior realimentação à vontade, proporcionou um crescimento rápido e maior que o do controle em, aproximadamente, 60 dias, diminuindo posteriormente, mas igualando-se a este ao final de 147 dias. O consumo de alimento foi alto até os 60 dias, mas diminuiuapós esse período, enquanto a taxa de crescimento específico e a eficiência de crescimento foram similares ao controle (Hayward \& Wang, 2001). Para Gasterosteus aculeatus, a aplicação de ciclos de 2, 4 e 6 dias de jejum seguidos por 2 dias de alimentação à vontade, durante 8 semanas, a $14^{\circ} \mathrm{C}$, mostrou que o consumo alimentar dos peixes foi maior no primeiro dia de realimentação e essa hiperfagia aumentou com a repetição dos ciclos, apresentando valores semelhantes ao controle para taxa de crescimento específico e eficiência de crescimento (Ali \& Wootton, 2001).

Durante o $3^{\circ}$ ciclo, tanto o peso quanto o comprimento e o fator de condição foram significativamente maiores $(\mathrm{P}<0,05)$ para os peixes do tratamento $\mathrm{A}$, em relação aos demais tratamentos. Respostas semelhantes foram 
observadas durante o $4^{\circ}$ ciclo, com diferenças significativas $(\mathrm{P}<0,05)$ entre os três tratamentos, para todas as variáveis analisadas.

Houve um crescimento mais acelerado dos peixes a partir de setembro, em todos os tratamentos, coincidindo com a elevação na temperatura da água (Figura 1). No $3^{\circ}$ ciclo, apesar de os peixes dos tratamentos $\mathrm{B}$ e $\mathrm{C}$ não terem conseguido alcançar o peso dos de A, eles expressaram o potencial para o crescimento compensatório, que coincidiu com o aumento na taxa de crescimento específico, eficiência de crescimento alta e conversão alimentar eficaz em todos os tratamentos. Adicionalmente, o fator de condição revelou que os peixes do tratamento A encontravam-se em melhores condições fisiológicas do que os demais.

O consumo de ração também aumentou nos períodos de temperatura da água mais elevada em todos os tratamentos (Figura 5). Angelini \& Petrere Jr. (1992) consideraram ótima a temperatura entre 25 e $27^{\circ} \mathrm{C}$ para o consumo pelo pacu, o que confirma as observações deste estudo.

Independentemente da manutenção da temperatura elevada, durante o $4^{\circ}$ ciclo alimentar, ocorreu um decréscimo na taxa de crescimento específico do pacu (Figura 3), inclusive no tratamento A. Respostas semelhantes foram observadas por Ferraz de Lima et al., (1988), que consideraram essa diminuição, provavelmente a partir de um tamanho entre 400 e $500 \mathrm{~g}$, como um fator limitante para o cultivo desta espécie.

A elevação da temperatura e, conseqüentemente, do metabolismo aumenta as exigências para sobrevivência, podendo ocorrer perdas de peso durante o jejum (Dobson \& Holmes 1984). No presente estudo, as altas temperaturas exigiram uma demanda extra de energia que os programas alimentares dos tratamentos B e C, no $4^{\circ}$ ciclo, não atenderam. Nestes tratamentos, as condições biológicas homeostáticas dos peixes foram excedidas para a recuperação do crescimento. A privação alimentar parece ter sido suficientemente severa, resultando em limitada capacidade de crescimento compensatório e/ou perda de peso do pacu, assim como observado em tilápia híbrida (Oreochromis mossambicus x O. niloticus) (Wang et al., 2000). Assim, a interação entre a duração e a severidade do período de restrição alimentar é de importância crítica para o sucesso do regime alimentar no restabelecimento do peso corporal (Jobling et al., 1993; Wang et al., 2000).

Sealey et al. (1998) relataram, para Ictalurus punctatus, crescimento compensatório em 9 semanas, utilizando um regime alimentar que alternava alimentação a cada 3, dias por 3 semanas, e realimentação de 3 semanas, durante 27 semanas, mas, após esse período, o peso do controle foi superior. Ciclos de um dia de jejum por semana proporcionaram um crescimento mais rápido em Oreochromis niloticus do que aqueles que foram alimentados diariamente, durante 24 semanas, a $27^{\circ} \mathrm{C}$ (Mélard et al., 1997). Ciclos de 2 e 14 dias de jejum com subseqüente realimentação, até o desaparecimento da hiperfagia, proporcionaram maior consumo de alimento e taxa de crescimento específico no híbrido Lepomis cyanellis x L. macrochirus, do que a alimentação ininterrupta (controle), a $24^{\circ} \mathrm{C}$, durante 105 dias. Entretanto, a maior eficiência de crescimento foi observada no grupo submetido a ciclo de 14 dias, em relação ao controle (Hayward et al., 1997). Após 10 semanas, a $28^{\circ} \mathrm{C}$, Ictalurus punctatus submetidos a ciclos alternados de 3 dias de privação alimentar e realimentação até o desaparecimento da hiperfagia, apresentaram maior peso corporal, maior consumo de alimento e melhor conversão alimentar, em relação ao controle, alimentado diariamente (Chatakondi \& Yant, 2001).

As evidências indicam que períodos mais curtos de privação alimentar sejam mais adequados do que períodos mais longos, para as espécies tropicais que apresentam intenso metabolismo e rápida taxa de

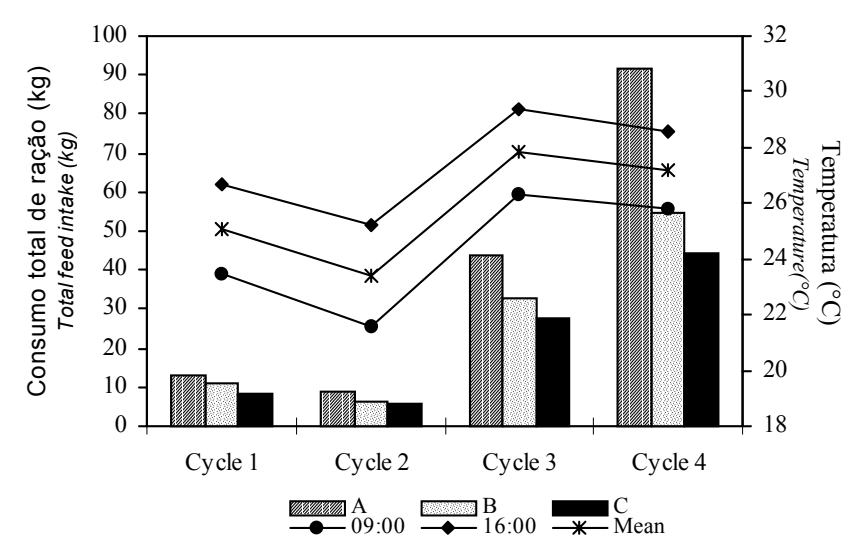

Figura 5 - Consumo total de ração pelos peixes por tratamento (A - alimentado ad libitum, diariamente; B - 4 semanas de restrição alimentar/9 semanas de realimentação; C - 6 semanas de restrição alimentar/7 semanas de realimentação), em cada ciclo alimentar.

Figure 5 - Total ration consumption in the treatments ( $A$ - control, fed daily ad libitum; $B$ - 4 weeks of feeding restriction/ 9 weeks of refeeding; $C$ - 6 weeks of feeding restriction/ 7 weeks of refeeding, in each cycle. 
crescimento (Mélard et al., 1997). Todavia, a possibilidade de que a capacidade para expressar o crescimento compensatório seja espécie específica e dependente da época do ano deve ser considerada (Hayward \&Wang 2001).

Apenas um peixe morreu (tratamento B) durante todo o período de estudo e a redução no número de peixes ( $\mathrm{n}$ - Tabela 3 ), a cada ciclo alimentar, ocorreu pela retirada de exemplares para a realização de outras análises (Souza et al., 2002).

\section{Avaliação econômica}

A produção obtida e os respectivos custos com alimentação, neste estudo, encontram-se nas Tabelas 3 e 4 .

O tratamento A apresentou menor biomassa e maior consumo de ração, quando comparado aos tratamentos $\mathrm{B}$ e $\mathrm{C}$, proporcionando uma receita líquida parcial 60,34 e $71,50 \%$ inferior aos respectivos tratamentos, durante o $1^{\circ}$ ciclo alimentar (Tabela 3 ). Para o $2^{\circ}$ ciclo, esses valores passaram a ser da ordem de 42,39 e $58,74 \%$ menores do que os tratamentos B e $\mathrm{C}$, respectivamente. Como o tratamento $\mathrm{C}$ resultou em maior biomassa, melhor conversão alimentar e maior receita líquida parcial durante as estações mais frias do ano, a economia na quantidade de ração utilizada durante os dois primeiros ciclos foi de, aproximadamente, $36,83 \%$ em relação ao A.

Nos demais ciclos alimentares, a resposta foi inversa, ou seja, a receita líquida parcial do tratamento A foi 14,03

Tabela 3 - Valores médios obtidos para biomassa total (BT), ganho em biomassa (GB), ração fornecida (RF), conversão alimentar aparente (CAA), custo da ração $(C R)$, custo com alimentação $(C A)$, receita bruta $(R B)$ e receita líquida parcial (RLP) do cultivo de pacu, por tratamento, em cada ciclo alimentar

Table 3 - Mean values of total biomass (TB), biomass gain (BG), offered ration (OR), apparent food conversion (AFC), ration cost (RC), feeding cost (FC), gross income (GI) and partial net income (PNI) in the pacu rearing, by treatment, in each feeding cycle

\begin{tabular}{|c|c|c|c|c|c|c|c|c|c|}
\hline & $\begin{array}{c}\text { Tratamentos } \\
\text { Treatments }\end{array}$ & $\begin{array}{c}\mathrm{BT}\left(\mathrm{kg} / 50 \mathrm{~m}^{2}\right) \\
T B \\
\end{array}$ & $\begin{array}{c}\mathrm{GB}\left(\mathrm{kg} / 50 \mathrm{~m}^{2}\right) \\
B G \\
\end{array}$ & $\begin{array}{c}\mathrm{RF}\left(\mathrm{kg} / 50 \mathrm{~m}^{2}\right) \\
\text { OR }\end{array}$ & $\begin{array}{l}\text { CAA } \\
A F C\end{array}$ & $\begin{array}{c}\mathrm{CR}(\mathrm{R} \$) \\
R C\end{array}$ & $\begin{array}{c}\mathrm{CA}(\mathrm{R} \$) \\
F C \\
\end{array}$ & $\begin{array}{c}\mathrm{RB}(\mathrm{R} \$) \\
G I\end{array}$ & $\begin{array}{c}\mathrm{RLP}(\mathrm{R} \$) \\
P N I\end{array}$ \\
\hline $1^{\circ}$ ciclo & A & 18,95 & 4,86 & 13,07 & 2,7 & 8,36 & 35,36 & 41,69 & 6,33 \\
\hline $1^{s t}$ cycle & B & 18,98 & 5,07 & 11,11 & 2,2 & 7,11 & 25,80 & 41,76 & 15,96 \\
\hline$(n=157)$ & $\mathrm{C}$ & 19,14 & 4,77 & 8,38 & 1,8 & 5,36 & 19,90 & 42,11 & 22,21 \\
\hline $2^{\circ}$ ciclo & A & 22,03 & 3,08 & 8,87 & 2,9 & 5,68 & 32,68 & 48,47 & 15,79 \\
\hline $2^{\text {nd }}$ cycle & B & 22,71 & 3,73 & 6,03 & 1,6 & 3,86 & 22,55 & 49,96 & 27,41 \\
\hline$(n=139)$ & $\mathrm{C}$ & 25,60 & 6,46 & 5,48 & 0,8 & 3,51 & 18,05 & 56,32 & 38,27 \\
\hline $3^{\circ}$ ciclo & A & 67,34 & 45,31 & 43,53 & 1,0 & 27,86 & 54,86 & 148,15 & 93,29 \\
\hline $3^{r d}$ cycle & B & 55,21 & 32,50 & 32,75 & 1,0 & 20,96 & 39,65 & 121,46 & 81,81 \\
\hline$(\mathrm{n}=121)$ & $\mathrm{C}$ & 54,35 & 28,75 & 27,43 & 1,0 & 17,56 & 32,10 & 119,55 & 87,45 \\
\hline $4^{\circ}$ ciclo & A & 115,24 & 47,90 & 91,79 & 1,9 & 58,75 & 85,75 & 253,53 & 167,78 \\
\hline $4^{\text {th }}$ cycle & B & 83,38 & 28,17 & 54,63 & 1,9 & 34,96 & 53,65 & 183,44 & 129,79 \\
\hline$(n=103)$ & $\mathrm{C}$ & 67,19 & 12,84 & 44,17 & 3,4 & 28,27 & 42,81 & 147,82 & 105,01 \\
\hline
\end{tabular}

A (controle, alimentado ad libitum, diariamente); B (4 semanas de restrição alimentar/9 semanas de realimentação); C (6 semanas de restrição alimentar/7 semanas de realimentação).

A (control, fed daily ad libitum); B (4 weeks of feeding restriction/9 weeks of refeeding); C (6 weeks of feeding restriction/7 weeks of refeeding).

Tabela 4 - Valores médios obtidos para biomassa total (BT), ganho em biomassa (GB), ração fornecida (RF), conversão alimentar aparente (CAA), custo da ração $(C R)$, custo com alimentação (CA), receita bruta (RB) e receita líquida parcial (RLP) do cultivo de pacu, por tratamento, durante o ano

Table 4 - Mean values of total biomass (TB), biomass gain (BG), offered ration (OR), apparent food conversion (AFC), ration cost (RC), feeding cost (FC), gross income (GI) and partial net income (PNI) in the pacu rearing, by treatment, during the year

\begin{tabular}{|c|c|c|c|c|c|c|c|c|}
\hline $\begin{array}{l}\text { Tratamentos } \\
\text { Treatments }\end{array}$ & $\begin{array}{c}\mathrm{BT}\left(\mathrm{kg} / 50 \mathrm{~m}^{2}\right) \\
T B \\
\end{array}$ & $\begin{array}{c}\mathrm{GB}\left(\mathrm{kg} / 50 \mathrm{~m}^{2}\right) \\
B G \\
\end{array}$ & $\begin{array}{c}\mathrm{RF}\left(\mathrm{kg} / 50 \mathrm{~m}^{2}\right) \\
O R\end{array}$ & $\begin{array}{l}\text { CAA } \\
A F C \\
\end{array}$ & $\begin{array}{c}\mathrm{CR}(\mathrm{R} \$) \\
R C \\
\end{array}$ & $\begin{array}{c}\mathrm{CA}(\mathrm{R} \$) \\
F C \\
\end{array}$ & $\begin{array}{c}\mathrm{RB}(\mathrm{R} \$) \\
G I\end{array}$ & $\begin{array}{c}\mathrm{RLP}(\mathrm{R} \$) \\
P N I \\
\end{array}$ \\
\hline A & 115,24 & 101,15 & 157,25 & 1,6 & 100,64 & 208,61 & 253,53 & 44,92 \\
\hline B & 83,38 & 69,47 & 104,53 & 1,5 & 66,90 & 141,66 & 183,44 & 41,78 \\
\hline $\mathrm{C}$ & 67,19 & 52,84 & 85,47 & 1,6 & 54,70 & 112,84 & 147,82 & 34,98 \\
\hline
\end{tabular}

A (controle, alimentado ad libitum, diariamente); B (4 semanas de restrição alimentar/9 semanas de realimentação); C (6 semanas de restrição alimentar/7 semanas de realimentação).

A (control, fed daily ad libitum); B (4 weeks of feeding restriction/9 weeks of refeeding); C (6 weeks of feeding restriction/7 weeks of refeeding).

\section{R. Bras. Zootec., v.32, n.1, p.19-28, 2003}


Tabela 5 - Valores projetados de biomassa total (BT), ganho em biomassa (GB), ração fornecida (RF), conversão alimentar aparente (CAA), custo da ração $(C R)$, custo com alimentação $(C A)$, receita bruta $(R B)$ e receita líquida parcial $(R L P)$ para o cultivo do pacu em ha/ano, por tratamento

Table 5 - Projects values of total biomass (TB), biomass gain (BG), offered ration (OR), apparent food conversion (AFC), ration cost (RC), feeding cost (FC), gross income (GI) and partial net income (PNI) in the pacu rearing, by treatment, in ha/year

\begin{tabular}{|c|c|c|c|c|c|c|c|c|}
\hline $\begin{array}{l}\text { Tratamentos } \\
\text { Treatments }\end{array}$ & $\begin{array}{c}\mathrm{BT}\left(\mathrm{kg} / 50 \mathrm{~m}^{2}\right) \\
T B\end{array}$ & $\begin{array}{c}\mathrm{GB}\left(\mathrm{kg} / 50 \mathrm{~m}^{2}\right) \\
B G\end{array}$ & $\begin{array}{c}\mathrm{RF}\left(\mathrm{kg} / 50 \mathrm{~m}^{2}\right) \\
O R\end{array}$ & $\begin{array}{l}\text { CAA } \\
A F C\end{array}$ & $\begin{array}{c}\mathrm{CR}(\mathrm{R} \$) \\
R C\end{array}$ & $\begin{array}{c}\mathrm{CA}(\mathrm{R} \$) \\
F C\end{array}$ & $\begin{array}{c}\mathrm{RB}(\mathrm{R} \$) \\
G I\end{array}$ & $\begin{array}{c}\mathrm{RLP}(\mathrm{R} \$) \\
P N I\end{array}$ \\
\hline A & 23.048 & 20.230 & 31.450 & 1,6 & $20.128,00$ & $21.423,84$ & $50.705,60$ & $29.281,76$ \\
\hline B & 16.676 & 13.894 & 20.906 & 1,5 & $13.379,84$ & $14.276,96$ & $36.687,20$ & $22.410,24$ \\
\hline $\mathrm{C}$ & 13.438 & 10.568 & 17.094 & 1,6 & $10.940,16$ & $11.637,92$ & $29.563,60$ & $17.925,68$ \\
\hline
\end{tabular}

A (controle, alimentado ad libitum, diariamente); B (4 semanas de restrição alimentar/9 semanas de realimentação); C (6 semanas de restrição alimentar/7 semanas de realimentação).

A (control, fed daily ad libitum); B (4 weeks of feeding restriction/9 weeks of refeeding); C (6 weeks of feeding restriction/7 weeks of refeeding).

e 6,68\% superior à dos tratamentos $\mathrm{B}$ e $\mathrm{C}$, no $3^{\circ}$ ciclo e, no $4^{\circ}$ ciclo, esses valores passaram a ser de $29,27 \mathrm{e}$ $59,78 \%$ superior aos respectivos tratamentos, apesar do maior consumo de ração (Tabela 3). Quando analisada a produção anual obtida para os três tratamentos (Tabela4), pode-se observar que a biomassa do tratamento A foi $71,51 \%$ superior à do $\mathrm{C}$ e, conseqüentemente, sua receita líquida parcial foi $28,42 \%$ superior à do referido tratamento.

Caso este cultivo tivesse sido projetado para uma área de 1 ha poderiam ser encontrados valores próximos aos da Tabela 5. Desta forma, a receita líquida parcial do tratamento A seria 30,66 e $63,35 \%$ superior à dos tratamentos $\mathrm{B}$ e $\mathrm{C}$.

\section{Conclusões}

Durante o período de outono/inverno, pode-seutilizar o ciclo alimentar de 6 semanas de restrição alternadas com 7 semanas de realimentação, no cultivo do pacu, sem que sua capacidade de expressar o crescimento compensatório seja prejudicada. Entretanto, durante as estações mais quentes do ano (primavera/verão), outros programas de alimentação devem ser testados, utilizandose períodos mais curtos de restrição alimentar, aliados à possibilidade da utilização de rações, cuja composição propiciem nutrientes melhor aproveitados pela espécie (Gonçalves, 2001), para dar o suporte energético necessário durante essa fase de maior crescimento e demanda metabólica.

\section{Agradecimento}

Às Rações Guabi Ltda, pela doação das rações; à J.L. Química da Água Ltda, pela doação dos reagentes utilizados nas análises de água; à Escola de
Veterinária da Universidade Federal de Goiás, pelo uso das instalações; e ao CNPq (Conselho Nacional de Desenvolvimento Científico e Tecnológico), pela concessão da bolsa de estudo.

\section{Literatura Citada}

ALI, M.; WOOTTON, R.J. Capacity for growth compensation in juvenile three-spined sticklebacks experiencing cycles of food deprivation. Journal of Fish Biology, v.58, p.1531-1544, 2001.

ANGELINI, R.; CANTELMO, O.A.; PETRERE JR., M. Determinação da taxa de consumo de ração pelo pacu Piaractus mesopotamicus Holmberg, 1887, com diferentes tamanhos e sob distintas temperaturas. Boletim Técnico do CEPTA, v.5, p.11-22, 1992.

ANGELINI, R.; PETRERE JR., M. Simulação da produção do pacu Piaractus mesopotamicus em viveiros de piscicultura. Boletim Técnico do CEPTA, v.5, p.41-55. 1992.

BANZATTO, D.A.; KRONKA, S.N. Experimentação agrícola. Jaboticabal: FUNEP, 1989. 247p.

BERNARDINO, G.; FERRARI, V.A. Efeitos do uso da ração comercial no desempenho do pacu, Piaractus mesopotamicus Holmberg, 1887 em cativeiro. Boletim Técnico do CEPTA, v.2, p.19-33, 1989 .

BLASCO, J.; FERNÁNDEZ, J.; GUTIÉRREZ, J. The effects of starvation and refeeding on plasma amimo acid levels, Cyprinus carpio L., 1758. Journal of Fish Biology, v.38, p.587-598, 1991.

BORGHETTI, J.R.; CANZI, C. The effect of water temperature and feeding rate on the growth rate of pacu (Piaractus mesopotamicus) raised in cages. Aquaculture, v.114, p.93-101, 1993.

CASTAGNOLLI, N.; CYRINO, J.E.P. Piscicultura nos trópicos São Paulo: Manole, 1986. 154p.

CHABALIN, E.; FERRAZDE LIMA, J.A. Análise econômica de um cultivo intensivo de pacu (Colossoma mitrei) no Centro-Oeste do Brasil. Boletim Técnico do CEPTA, v.1, p.1-68, 1988.

CHATAKONDI, N.G.; YANT, R.D. Application of compensatory growth to enhance production in channel catfish Ictalurus punctatus. Journal of World Aquaculture Society, v.32, n.3, p.278-285, 2001.

DOBSON, S.H.; HOLMES, R.M. Compensatory growth in the rainbow trout, Salmo gairdneri Richardson. Journal of Fish Biology, v.25, p.649-656, 1984.

EINEN, O.; WAAGAN, B.; THOMASSEN, M.S. Starvation prior to slaughter in Atlantic salmon (Salmo salar). I. 
Effects on weight loss, body shape, slaughter-and filletyield, proximate and fatty acid composition. Aquaculture, v.166, p.85-104, 1998.

FERRARI, V.A.; LUCAS, A.F.B.; GASPAR, L.A. Monocultura de pacu Piaractus mesopotamicus Holmberg, 1887, em condições de viveiro-estufa. Boletim Técnico do CEPTA, v.3, p.33-42, 1990.

FERRAZ DELIMA, J.A.; FERRARI, V.A.; COLARES DE MELO, J.S. et al. Comportamento do pacu, Colossoma mitrei, em cultivo experimental, no Centro-Oeste do Brasil. Boletim Técnico do CEPTA, v.1, p.15-28, 1988.

FIGUEROA， R.I.; RODRÍGUEZ-SABARÍS， R.; ALDEGUNDE, M. et al. Effects of food deprivation on 24 h-changes in brain and liver carbohydrate and ketone body metabolism of rainbow trout. Journal of Fish Biology, v.57, p.631-646, 2000.

GONÇALVES, F.D. Metabolismo energético e desempenho produtivo de juvenis de pacu (Piaractus mesopotamicus), submetidos a jejum e realimentação com dietas contendo diferentes níveis de carboidrato e proteína. Jaboticabal: Universidade Estadual Paulista. 2001. 67p. Dissertação (Mestrado em Aqüicultura em Águas Continentais) - Universidade Estadual Paulista, 2001.

HAYWARD, R.S.; NOLTIE, D.B.; WANG, N. Use of compensatory growth to double hybrid sunfish growth rates. Transaction of the American Fisheries Society, v.126, p.316-322, 1997.

HAYWARD, R.S.; WANG, N. Failure to induce over-compensation of growth in maturing yellow perch. Journal of Fish Biology, v.59, p.126-140, 2001.

HAYWARD, R.S.; WANG, N.; NOLTIE, D.B. Group holding impedes compensatory growth of hybrid sunfish. Aquaculture, v.183, p.299-305, 2000.

HUNG, S.S.O.; LIU, W.; LI, H. et al. Effect of starvation on some morphological and biochemical parameters in while sturgeon, Acipenser transmontanus. Aquaculture, v.151, p.357-363, 1997.

JOBLING, M.; JØRGENSEN, E.H.; SIIKAVUOPIO, S.I. The influence of previous feeding regime on the compensatory growth response of maturing and immature arctic charr, Salvelinus alpinus. Journal of Fish Biology, v.43, p.409-419, 1993.

KIEFFER, J.D.; TUFTS, B.L. Effects of food deprivation on white muscle energy reserves in rainbow trout (Oncorhynchus mykiss): the relationships with body size and temperature. Fish Physiology and Biochemistry, v.19, p.239-245, 1998.

MACLEAN, A.; METCALFE, N.B. Social status, access to food, and compensatory growth in juvenile Atlantic salmon. Journal of Fish Biology, v.58, p.1331-1346, 2001.

MEHNER, T.; WIESER, W. Energetic and metabolic correlates of starvation in juvenile perch (Percafluviatilis). Journal of Fish Biology, v.45, p.325-333, 1994.

MÉLARD, C.; BARAS, E.; DESPREZ, D. Compensatory growth of Nile tilapia Oreochromis niloticus. In: INTERNATIONAL SYMPOSIUM ON TILAPIA IN AQUACULTURE 4, vol. 1, 1997, Orlando. Proceedings... Orlando: NRAES, 1997. p.178-185.

OSTRENSKY, A.; BOEGER, W. Piscicultura: fundamentos e técnicas de manejo. Guaíba: Agropecuária, 1998. 211p.

PAUL, A.J.; PAUL, J.M.; SMITH, R.L. Compensatory growth in Alaska yellowfin sole, Pleuronectes asper, following food deprivation. Journal of Fish Biology, v.46, p.442-448, 1995.

PETRERE JR., M. River fisheries in Brazil: a review. Regulated Rivers Research \& Management, v.4, p.1-16, 1989.

QIAN, X.; CUI, Y.; XIONG, B.; YANG, Y. Compensatory growth, feed utilization and activity in gibel carp, following feed deprivation. Journal of Fish Biology, v.56, p.228-232, 2000.

QUINTON, J.C.; BLAKE, R.W. The effect of feed cycling and ration level on the compensatory growth response in rainbow trout, Oncorhynchus mykiss. Journal of Fish Biology, v.37, p.33-41, 1990.

RICKER, W.E. Computation and interpretation of biological statistics of fish populations. Bulletin of the Fisheries Research Board Canada, v.191, 382p. 1975.

SEALEY, W.M.; DAVIS, J.T.; GATLIN III, D.M. Restricted feeding regimes increase production efficiency in channel catfish. Auburn: Southern Regional Aquaculture Center, 1998. 5p. (SRAC Publication, 189).

SILVA, A.J. Aspectos de alimentação do pacu adulto, Colossoma mitrei (Berg, 1985) (Pisces, Characidae), no pantanal de Mato Grosso. Rio de Janeiro: Universidade Federal do Rio de Janeiro, 1985. 92p. Dissertação (Mestrado em Ciências Biológicas) - Universidade Federal do Rio de Janeiro, Rio de Janeiro, 1985.

SIPAÚBA-TAVARES, L.H. Limnologia aplicada à aqüicultura. Jaboticabal: FUNEP, 1994. 70p.

SIROL, R.N.; SALARO, A.L.; ANDRADE, D.R. Diferentes condições nutricionais de alevinos de tilápia vermelha (Oreochromis niloticus x O. aureus), submetidos à inanição. In: SIMPÓSIO BRASILEIRO DE AQÜICULTURA, 11., 2000, Florianópolis. Anais... Florianópolis: ABRAq, 2000, não paginado, CD-ROM. Artigos. Nutrição - 12.

SOUZA, V.L. Efeitos da restrição alimentar e da realimentação no crescimento e metabolismo energético de juvenis de pacu (Piaractus mesopotamicus Holmberg, 1887). Jaboticabal: Centro de Aqüicultura da Universidade Estadual Paulista, 1998. 118p. Tese (Doutorado em Aqüicultura) - Centro de Aqüicultura da Universidade Estadual Paulista, 1998.

SOUZA, V.L.; OLIVEIRA, E.G.; URBINATI, E.C. Effects of food restriction and refeeding on energy stores and growth of pacu, Piaractus mesopotamicus (Characidae). Journal of Aquaculture in the Tropics, v.15, n.4, p.371-379, 2000.

SOUZA, V.L.; URBINATI, E.C.; GONÇALVES, D.C.; SILVA, P.C. Composição corporal e índices biométricos do pacu, Piaractus mesopotamicus, 1887 (Osteichthyes, Characidae) submetido a ciclos alternados de restrição alimentar e realimentação. Acta Scientiarum, v. 24, n. 2, p.533-540, 2002.

WANG, Y; CUI, Y.; YANG, Y.; CAI, F. Compensatory growth in Hybrid tilapia, Oreochromis mossambicus $x$ O. niloticus, reared in sea water. Aquaculture, v.189, p.101-108, 2000.

WEATHERLEY, A.H.; GILL, H.S. The biology of fish growth. London: Academic Press, 1987. 443p.

WIESER, W.; KRUMSCHNABEL, G.; OJWANG-OKWOR, J.P. The energetic of starvation and growth after refeeding in juveniles of three cyprinid species. Environmental Biology Fishes, v.33, p.63-71, 1992.

XIE, S.; ZHU, X.; CUI, Y. et al. Compensatory growth in the gibel carp following feed deprivation: temporal patterns in growth, nutrient deposition, feed intake and body composition. Journal of Fish Biology, v.58, p. 999-1009, 2001.

ZAMAL, H.; OLLEVIER, F. Effect of feeding and lack of food on the growth, gross biochemical and fatty acid composition of juvenile catfish. Journal of Fish Biology, v.46, p.404414, 1995.

Recebido em: 01/02/02 Aceito em: 12/09/02

R. Bras. Zootec., v.32, n.1, p.19-28, 2003 